\title{
Emergency endoscopic third ventriculostomy for blocked shunts? Univariate and multivariate analysis of independent predictors for failure
}

\author{
David Y. C. Chan, MRCS, Anderson C. O. Tsang, MRCS, Wilson W. S. Ho, FRCS, LLB, MBA, \\ Kevin K. F. Cheng, FRCS, Lai F. Li, FRCS, Frederick C. P. Tsang, FCSHK, \\ Benedict B. T. Taw, FRCS, Jenny K. S. Pu, FRCS, MS, \\ Gilberto K. K. Leung, FRCS, MS, PhD, LLM, and Matthew W. M. Lui, FRCS
}

Division of Neurosurgery, Department of Surgery, Li Ka Shing Faculty of Medicine, The University of Hong Kong, Queen Mary Hospital, Hong Kong

\begin{abstract}
OBJECTIVE Hydrocephalus with a blocked ventriculoperitoneal (VP) shunt is a life-threatening condition. Emergency endoscopic third ventriculostomy (ETV) is a potential treatment option. The aim of the study was to identify independent risk factors associated with failure of ETV in treating patients with blocked shunts.

METHODS The authors retrospectively reviewed data from consecutive patients admitted for blocked shunt treated by ETV during the study period from 2000 to 2016 . Univariate and multivariate analyses were performed to identify independent factors associated with failed ETV for blocked shunts, such as age, sex, history of CNS infection, number of previous shunt revisions, operations performed as an emergency or elective, number of specialists, and other factors.

RESULTS In total, 121 patients underwent ETV during the study period. Of these, 31 patients (25.6\%) had ETV for treatment of a blocked shunt. In $25(80.6 \%)$ of 31 ETV was performed as an emergency procedure. There was no significant difference in the success rate of ETV depending on whether it was performed as an emergency procedure (64\% [16/25]) or an elective procedure (66.7\% [4/6]; OR $0.062,95 \% \mathrm{Cl} 0.001-2.708 ; p=0.149)$. Univariate and multivariate analyses identified that history of a CNS infection was an independent risk factor for failure of ETV in treating patients with a blocked shunt (OR $0.030,95 \% \mathrm{Cl} 0.001-0.888 ; p=0.043)$.
\end{abstract}

CONCLUSIONS Emergency ETV had a comparable success rate as elective ETV. A history of CNS infection is an independent predictor of ETV failure in treating patients with blocked shunts.

https://thejns.org/doi/abs/10.3171/2018.6.JNS1865

KEYWORDS endoscopic third ventriculostomy; hydrocephalus; shunt malfunction; ventriculoperitoneal shunt

$\mathrm{H}$ YDROCEPHALUS with a blocked ventriculoperitoneal (VP) shunt is a life-threatening condition. Treatment options include shunt revision or externalization of the shunt. Pitfalls include risks of further shunt failure from either a blocked shunt or shunt infection. An alternative treatment is emergency endoscopic third ventriculostomy (ETV).

The ETV success score (ETVSS) has been shown to have good predictive value in the treatment's success rate. ${ }^{15,17}$ However, an external validation study has shown that the predictive value of ETVSS for secondary ETV is not as accurate as that for primary ETV.${ }^{19}$ In fact, the shortcoming of the ETVSS model was identified to have overestimated the success rate in patients having secondary ETV, such as those patients with a preexisting shunt. ${ }^{19}$ Hence, there is still a knowledge gap when it comes to selecting the appropriate patients with blocked shunts for ETV.

The aim of the study was to evaluate the treatment outcome of ETV in patients with blocked VP shunts. At the same time, this study aimed, using a logistic regression model, to identify independent risk factors associated with failure of ETV when treating patients with a blocked shunt. 


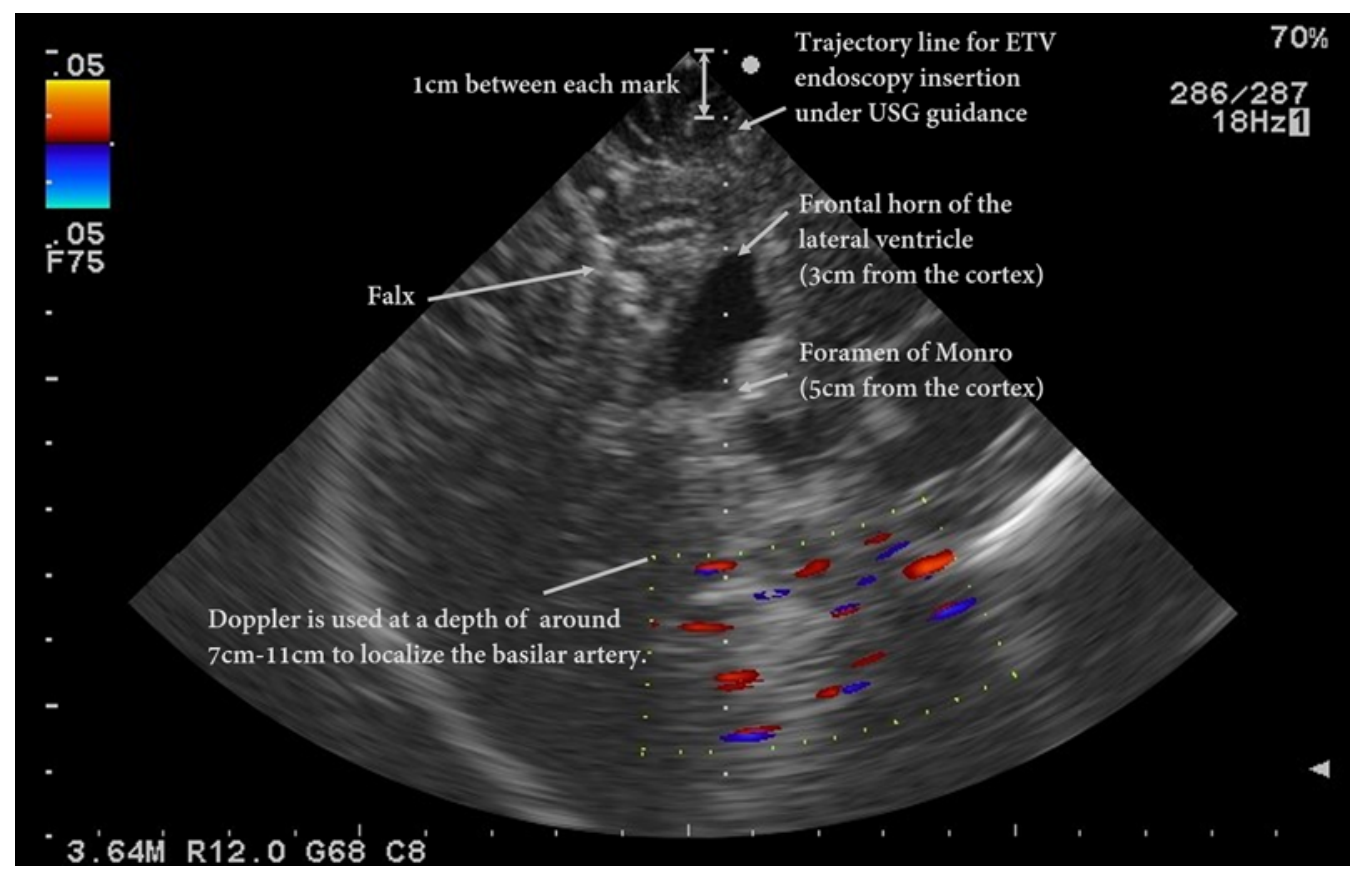

FIG. 1. USG was used to guide insertion of the ETV endoscope. The USG probe can identify an optimum trajectory by aligning the foramen of Monro to the floor of the third ventricle. If the alignment is suboptimal, more bone drilling can be performed before dural opening. The Doppler function of the USG was used as well to detect the site of the basilar artery (not shown in this plane). This can determine the angle of the trajectory in the sagittal plane for endoscope insertion. Figure is available in color online only.

\section{Methods}

We retrospectively reviewed consecutive patients admitted to the Department of Neurosurgery, Queen Mary Hospital, Hong Kong, during the study period from 2000 to 2016. All patients had undergone preoperative and postoperative neurological and radiological evaluation. Inclusion criteria were as follows: 1) patients admitted with preexisting shunt(s), 2) index admission was a result of shunt failure/blocked shunt, 3) patients were treated by ETV during the same admission, and 4) patients were with or without simultaneous shunt removal during the same operation. The exclusion criterion was if ETV for the blocked shunt was performed at another hospital.

Successful ETV treatment was defined as no clinical signs and symptoms of raised intracranial pressure requiring reoperation or shunting.

Emergency operations are defined as procedures arranged upon the patient's unscheduled admission to the hospital via emergency department due to symptoms of raised intracranial pressure from hydrocephalus as a result of shunt failure. These patients were judged by the on-call neurosurgeons to be in unstable condition and in need of urgent treatment by CSF diversion.

Elective operations are defined as procedures arranged in scheduled elective operation lists. These patients were judged by the admitting neurosurgeons to be in relatively stable condition without the immediate need for CSF diversion.

\section{Surgical Procedure}

For the surgical procedure, there were two types of positioning depending on the entry site of the preexisting shunt. For those with shunts placed via a frontal burr hole, patients were placed in the supine position and their heads were placed in a neutral position. The ETV entry site was either through the ipsilateral frontal wound (after enlarging the preexisting frontal burr hole) or through a new contralateral frontal burr hole. For those shunts placed via a parietal burr hole, patients had shoulder support and their heads were kept in a lateral position. This allowed exposure of both the frontal and parietal regions of the ipsilateral side at the same time. The occipital scalp wound was made to expose and retrieve the old preexisting VP shunt. A new ipsilateral frontal scalp wound and the burr hole were fashioned at the coronal suture for the entry of the endoscope for third ventriculostomy.

Ultrasonography (USG) was used for guidance of insertion of the endoscope. A mini-craniotomy of 1 inch in diameter was created to allow simultaneous placement of both the USG probe and the endoscopy trocar at the cortical surface. The USG probe could then identify an optimum entry site by aligning the foramen of Monro with the floor of the third ventricle. If the alignment was suboptimal, more bone drilling could be performed before dural opening. The Doppler function of the USG was used as well to detect the site of the basilar artery (Fig. 1).

After the insertion of the endoscope into the ipsilateral lateral ventricle, the preexisting shunt could be identified from the endoscopic view (Fig. 2). Adhesions could be dissected out from the side holes of the ventricular catheter tip directly under endoscopic visualization. This allowed for a safer removal of the old shunt's ventricular catheter, and direct endoscopic hemostasis could be performed if 


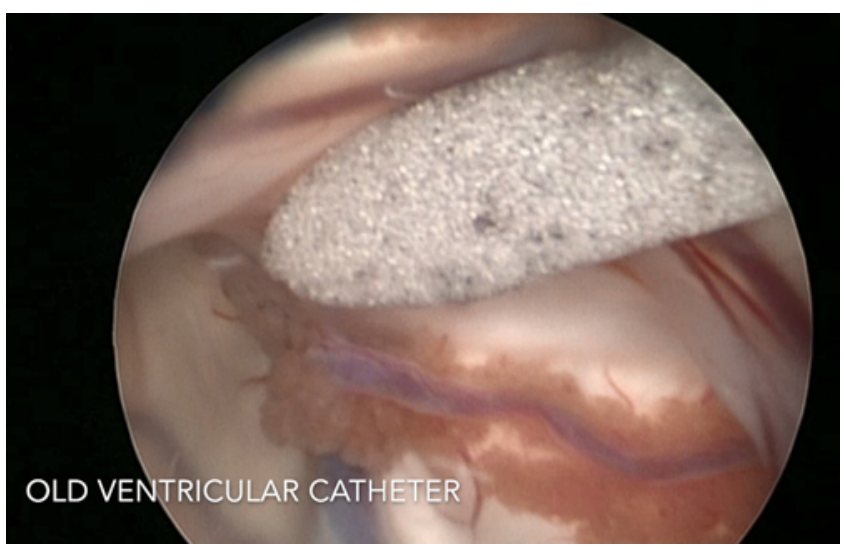

FIG. 2. After insertion of the endoscope into the lateral ventricle, the preexisting shunt can be identified from the endoscopic view. Adhesions can be dissected out from the side holes of the ventricular catheter tip directly under endoscopic visualization. This allows for safer removal of the old shunt's ventricular catheter, and direct endoscopic hemostasis can be performed if necessary. Figure is available in color online only.

necessary. In contrast to the conventional supine position, the endoscopic view of the floor of the third ventricle was turned $90^{\circ}$ for patients in the lateral position (Fig. 3). The ventriculostomy was performed at the floor of the third ventricle with forceps and neuroballoon.

\section{Statistical Analysis}

Univariate and multivariate analyses were performed to identify independent factors associated with failed ETV for blocked shunts. Variables considered in the statistical analysis included sex, age at the time of ETV, age at the time of the initial VP shunt placement, type of hydrocephalus (obstructive or communicating), history of CNS infection, number of previous shunt revisions, and interval between the initial VP shunt surgery and ETV. Operative factors included whether surgery was performed as an emergency or elective procedure, the time during the day the operation was conducted (either daytime from $8 \mathrm{Am}$ to 6 PM or night shift from 6 PM to $8 \mathrm{AM}$ ), number of specialists involved, the duration of the operation, the entry site and laterality of the preexisting old shunt, the entry site of the new ETV endoscope, the positioning for the ETV, and the use of USG as guidance for insertion of the endoscope.

Statistical analysis was performed with the chi-square test, Fisher's exact test, and unpaired t-test. Univariate analysis was performed with the general linear model. Multivariate analysis was performed with logistic regression. For the multivariate model, we selected a priori covariates based on variables used in previous publications, ${ }^{15,16}$ such as age, the presence of VP shunts, type of hydrocephalus, and history of infection. Specific exposure and outcomes for evaluation were included, such as type of operation (emergency or elective) and the number of specialists. The corresponding odds ratio with $95 \% \mathrm{CI}$ was included with significance set at $\mathrm{p}=0.05$. Statistical analysis was performed with the Statistical Package for the Social Sciences for Microsoft Windows (SPSS version 24.0.0.).

Ethics approval was obtained from the Institutional

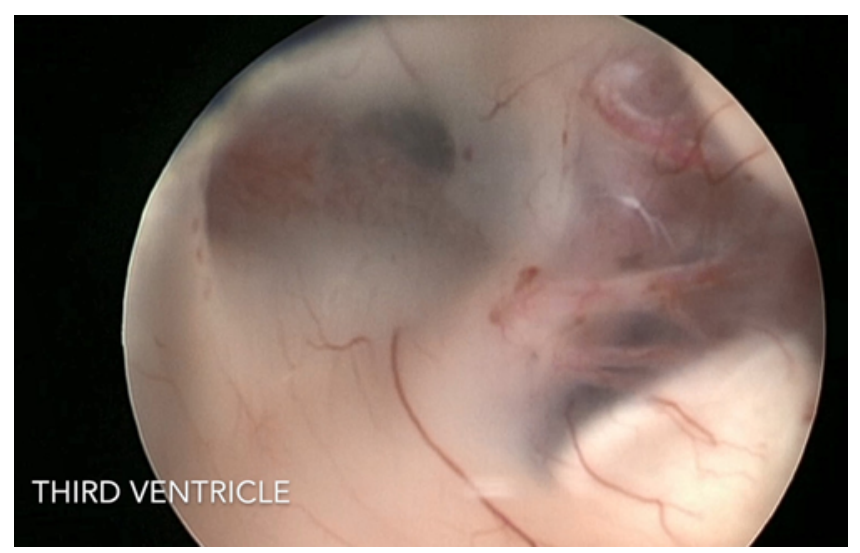

FIG. 3. In contrast to the conventional ETV performed with the patient in the supine position, the endoscopic view of the floor of the third ventricle was turned $90^{\circ}$ for patients in the lateral position. In this case, ETV and parietal shunt removal can be performed at the same time. Figure is available in color online only.

Review Board of the University of Hong Kong/Hospital Authority Hong Kong West Cluster.

\section{Results}

In total, 121 consecutive patients underwent ETV during the study period. Of these, 31 patients $(25.6 \%)$ underwent ETV for the treatment of a blocked shunt during the study period. For the background demographics, the average age of the patients was 23.6 years (range $0-60$ years). The average duration of follow-up was 5.4 years (range 1.5-16 years). As to the category of operation, $80.6 \%$ (25/31) were performed as emergency procedures, and $19.4 \%(6 / 31)$ were performed as elective procedures.

The median time from admission to emergency surgery was 12 hours 3 minutes (range 3 hours 46 minutes to 25 hours). The median time from admission to elective surgery was 25 hours (range 14 hours to 54 days). The interval between admission and surgery for this group of patients did not reflect the clinical situation, as some of the patients were preterm infants with intraventricular hemorrhage. They remained as inpatients after VP shunt insertion and subsequently received ETV during the same hospital admission.

Tables 1 and 2 show the results of the univariate analysis on the risk factors associated with the success of ETV. Of those who had a successful ETV, the average age had no significant impact on those in whom ETV failed in cases of a blocked shunt (27.05 years [ETV success] vs 20.64 years [ETV failure]; $\mathrm{p}=0.334$ ). At the same time, there was no significant difference in the age of the patients at the time of VP shunt placement. Those with successful ETV had their corresponding initial VP shunt surgery performed during adulthood at an average age of 21.37 years as compared with those with ETV failure who had their VP shunt surgery performed during childhood, at an average age of 13.6 years $(p=0.183)$. The interval between the initial VP shunt insertion and ETV was shorter for those with successful ETV, although this was not statistically significant $(\mathrm{p}=0.592)$. 
TABLE 1. Univariate analysis of patient factors related to successful ETV for treatment of blocked shunts

\begin{tabular}{lccc}
\hline \multicolumn{1}{c}{ Factor } & Successful ETV $(\mathrm{n}=20)$ & Failed ETV Requiring Shunting $(\mathrm{n}=11)$ & $\mathrm{p}$ Value \\
\hline Mean age (range), yrs & $27.05(0-60)$ & $20.64(0-46)$ & 0.344 \\
\hline Age at ETV & & & 0.552 \\
\hline$<1$ yr & $0(0)$ & $2(18.2)$ & $2(18.2)$ \\
\hline $1-10$ yrs & $4(20)$ & $7(63.3)$ & 0.401 \\
\hline$>10$ yrs & $16(80)$ & $4(36.4)$ & 0.183 \\
\hline Female sex & $13(65)$ & $13.36(0-39)$ & 0.602 \\
\hline Mean age at initial VP shunting (range), yrs & $21.37(0-40)$ & $8(72.7)$ & 0.123 \\
\hline Initial VP shunt placed in childhood, no. (\%) & $11(55)$ & $4(36.4)$ & $0.014^{*}$ \\
\hline Presence of obstructive hydrocephalus, no. (\%) & $6(30)$ & $6(54.5)$ & \\
\hline History of CNS infection, no. (\%) & $2(10)$ & $2(18.2)$ & $5(45.5)$ \\
\hline No. of previous shunt revisions & $6(30)$ & $4(36.4)$ & \\
\hline 0 & $10(50)$ & $7.28 \pm 2.33$ & 0.594 \\
\hline 1 & $4(20)$ & & \\
\hline$\geq 2$ & $5.76 \pm 1.65$ & & \\
\hline Mean interval btwn initial VP shunt \& ETV op, yrs & & \\
\hline Values are presented as the number (\%) of patients, the mean (range), or mean \pm SD. &
\end{tabular}

Of 31 patients, 19 had their initial shunt placed during childhood. Twelve of these 19 pediatric shunt patients had no history of infection. There were 8 patients with a history of CNS infection: 2 had meningitis with positive CSF culture growth with bacillus, and 6 had a history of shunt infection (4 had coagulase-negative Staphylococcus and 2 had Enterococcus infections). All the cases were treated with antibiotics followed by VP shunt placement. None of the patients in this study had active CNS infection at the time of ETV. The success rate of ETV was $75 \%(9 / 12)$ in patients whose shunts were placed during childhood with no history of infection. This was significantly higher than the $28.6 \%(2 / 7)$ success rate for patients with a pediatric shunt and history of infection ( $\mathrm{p}=0.048)$.

Dissection of adhesions was performed for VP shunt removal. Of the 31 cases in this study, 23 underwent removal of the complete VP shunt system and 8 underwent removal of ventricular catheters and shunt valves, followed by ligation of peritoneal catheters. All 31 patients had the ventricular catheter of the VP shunt removed. Hence, CSF diversion was a result of successful ETV rather than functional VP shunts after dissection of adhesions.

Eleven patients had post-ETV persistent hydrocephalus requiring reoperations. The median interval between failed ETV and reoperation was 8 days. Nine patients were treated with a new VP shunt. One had craniotomy for fenestration. One patient was treated with another ETV that was successful, and the patient remained shunt free. There was no mortality.

The success rate of emergency ETV was comparable to that of elective ETV for treatment of a blocked shunt. The success rate of ETV for emergency surgery was $64 \%$ $(16 / 25)$ compared to that of $66.7 \%$ (4/6) for elective operations (OR 0.062, 95\% CI 0.001-2.708; $\mathrm{p}=0.149$ ).

In $32.3 \%$ (10/31) of patients, surgeries were performed by 1 specialist with 1 trainee. In $48.4 \%$ (15/31), surgeries were performed by 2 specialists. In 19.4\% (6/31), surgeries were performed by 3 or more specialists. There was no significant difference in the success rate according to whether the procedure was conducted by one specialist versus two or more specialists. However, there could be selection bias in that procedures performed by one specialist might have been less complicated.

In $38.7 \%$ (12/31) of the cases, USG was used for guidance of endoscope insertion for ETV. In fact, all cases utilizing USG were emergency ETVs. In some cases of emergency ETV, USG was not used. In those cases, the preexisting VP shunt was at the frontal burr hole, and insertion of the endoscope followed the old track at the ipsilateral burr hole immediately after shunt removal.

Table 3 showed the results of multivariate logistic regression analysis. It identified a history of CNS infection as an independent risk factor for failure of ETV in treating patients with a blocked shunt (OR 33.3, 95\% CI 1.125$1000 ; p=0.043$ ).

\section{Discussion}

As hydrocephalus with a blocked VP shunt is a lifethreatening condition, emergency surgery is the treatment of choice. The role of ETV for the treatment of a blocked shunt has been established, but, in general, this practice has not been widely adopted in the emergency setting. In their case series, Boschert et al. reported that 6 of 17 (35.3\%) ETV procedures performed to treat a blocked shunt were performed as emergency operations; ${ }^{2}$ otherwise, most studies have not specifically reported whether ETV was performed as an emergency procedure. There is a knowledge gap on the potential hurdles in performing ETV as an emergency operation; hurdles include availability of expertise, lack of instruments, and inadequacy of preoperative workup. As temporary measures, an external ventricular drain has been inserted to relieve hydrocephalus in an emergency setting. ${ }^{18}$ However, it is not ideal 
TABLE 2. Univariate analysis of operative factors related to successful ETV for treatment of blocked shunts

\begin{tabular}{|c|c|c|c|}
\hline Factor & $\begin{array}{l}\text { Successful } \\
\text { ETV }(\%)\end{array}$ & $\begin{array}{c}\text { Failed ETV Requiring } \\
\text { Shunting }(\%)\end{array}$ & $\begin{array}{c}p \\
\text { Value }\end{array}$ \\
\hline No. of patients & 20 & 11 & \\
\hline Type of op & & & 0.330 \\
\hline Elective & $4(20)$ & $2(18.2)$ & \\
\hline Emergency & $16(80)$ & $9(81.8)$ & \\
\hline Timing of op & & & 0.654 \\
\hline Daytime (0800-1800) & $11(55)$ & $8(72.7)$ & \\
\hline Night shift (1800-0800) & $9(45)$ & $3(27.3)$ & \\
\hline No. of specialists & & & 0.515 \\
\hline 1 & $7(35)$ & $3(27.3)$ & \\
\hline$\geq 2$ & $13(65)$ & $8(72.7)$ & \\
\hline Mean op duration, mins & $95.6 \pm 15.8$ & $95.5 \pm 14.5$ & 0.997 \\
\hline Entry site of old shunt & & & 0.981 \\
\hline Frontal & $9(45)$ & $5(45.5)$ & \\
\hline Parietal & $11(55)$ & $6(54.5)$ & \\
\hline Laterality of old shunt & & & 0.809 \\
\hline Right & $10(50)$ & $5(45.5)$ & \\
\hline Left & $10(50)$ & $6(54.5)$ & \\
\hline Entry site of new ETV & & & 0.975 \\
\hline Right frontal & $15(75)$ & $7(63.6)$ & \\
\hline Left frontal & $5(25)$ & $4(36.4)$ & \\
\hline Op position for ETV & & & 0.860 \\
\hline Supine & $11(55)$ & $7(63.6)$ & \\
\hline Lateral & $9(45)$ & $4(36.4)$ & \\
\hline $\begin{array}{l}\text { Use of USG for ETV } \\
\text { endoscope insertion }\end{array}$ & $6(30)$ & $6(54.5)$ & 0.113 \\
\hline
\end{tabular}

USG = ultrasonography.

Values are presented as the number (\%) of patients. Mean values are presented \pm SD.

for pediatric patients to undergo two separate exposures to general anesthesia. In contrast to previous studies, our study specifically examined the factors that could potentially hinder the execution of ETV as an emergency operation, such as time of the day the procedure was performed (daytime versus night shift), number of specialists involved in the procedure, location of the preexisting shunt (such as parietal region), and positioning for the patient (such as lateral), in addition to analyzing patient factors such as history of infection and type of hydrocephalus.

Given that the majority of the patients in this study initially received a shunt in childhood, this article can be seen to relate to the question of "transition of care" from pediatric to adult neurosurgical care. Age was shown to be a major determinant of the success of ETV, and younger patients had a poorer outcome. ${ }^{6}$ The ETV success score (ETVSS) had age as a main factor in predicting the treatment outcome of ETV. ${ }^{16}$ This score has been shown to have good value in predicting the treatment success rate of ETV in general. ${ }^{15,17}$ However, for secondary ETV-i.e., ETV for patients with preexisting shunts-an external validation study has shown that the predictive value of ETVSS was not as accurate for primary ETV..$^{19}$ In fact, one shortcom-
TABLE 3. Multivariate logistic regression analysis of factors related to successful ETV for the treatment of blocked shunts

\begin{tabular}{lrll}
\hline \multicolumn{1}{c}{ Factor } & OR & \multicolumn{1}{c}{$95 \% \mathrm{Cl}$} & $\mathrm{p} \mathrm{Value}$ \\
\hline Age & 2.008 & $0.056-71.974$ & 0.703 \\
\hline Sex & 10.964 & $0.253-475.409$ & 0.213 \\
\hline Initial VP shunt placed in childhood & 0.820 & $0.006-118.835$ & 0.938 \\
\hline $\begin{array}{l}\text { Obstructive or communicating } \\
\text { hydrocephalus }\end{array}$ & 18.920 & $0.370-967.35$ & 0.143 \\
\hline History of CNS infection & 0.030 & $0.001-0.888$ & $0.043^{*}$ \\
\hline No. of specialists & 0.081 & $0.002-3.347$ & 0.186 \\
\hline Elective or emergency op & 0.062 & $0.001-2.708$ & 0.149 \\
\hline No. of shunt revisions & 0.671 & $0.113-3.993$ & 0.661 \\
\hline
\end{tabular}

* Statistically significant.

ing of the ETVSS model was that it overestimated the success rate in patients undergoing secondary ETV, such as patients with preexisting shunts. ${ }^{19}$ A good outcome of ETV for shunt malfunction in previously shunt-treated infants has been reported. ${ }^{1}$ Our study has concordant findings: ETV was shown to have a high success rate in patients whose shunt was placed during childhood as long as there was no history of CNS infection.

ETV was initially shown to be effective for the treatment of shunt dysfunction in patients with occlusive hydrocephalus. ${ }^{2}$ In this type of case in which patients were previously shunt dependent, CSF absorptive capacity was shown to be gradually improved within 1-2 weeks after ETV. ${ }^{21}$ ETV was also reported to be effective in the treatment of shunt infection by allowing removal of all cranial implants. ${ }^{11}$ As a result, ETV was proposed to be the firstline therapeutic option for the treatment of a blocked shunt in cases of initial obstructive hydrocephalus. ${ }^{4,5}$ In this case, these patients could have an opportunity of becoming shunt independent. ${ }^{25}$

At the same time, ETV has been shown to be effective in treating cases of communicating hydrocephalus. ${ }^{8,9,12}$ The success rate of ETV for shunt malfunction was reported to be not specific to the original etiology. ${ }^{22}$ The success rate of secondary ETV for nonobstructive hydrocephalus has been shown to be comparable to that of primary ETV.22 For patients who undergo ETV to treat shunt malfunction, the success rate has tended to be higher with shunt ligation. ${ }^{20}$ Simultaneous shunt removal in the same setting as ETV has also been reported with good outcomes..$^{10,14}$

We acknowledged the distinction between different types of hydrocephalus is not always so clear. In the present study, the diagnosis of patients with obstructive hydrocephalus was clear-cut, including thalamic tumors, ventricular tumors, midbrain tumors, pineal tumors, and brainstem cavernomas. There were dilatation of the lateral ventricles and the third ventricle, while the fourth ventricle was small in these cases. For communicating hydrocephalus, the majority of patients in this study were diagnosed with neonatal intraventricular hemorrhage or meningitis. There was one patient with achondroplasia and one with Pfeiffer syndrome. Dilatation of the fourth ventricle was present as well in these cases. We acknowledge there were cases with obstruction beyond the ventricles, such as extra- 
ventricular intracisternal hydrocephalus.,12 Overall, there was no significant difference in the ETV success rate between the two types of hydrocephalus in this study. The results of this study show that a certain proportion of patients with communicating hydrocephalus and blocked VP shunts can be successfully treated with ETV. With a cohort of 31 patients, our analysis may be underpowered to detect differences in the success rate of ETV in an emergency operation versus an elective operation.

ETV can be facilitated by the use of USG guidance for insertion of the endoscope. ${ }^{23}$ It can also be enhanced with the use of CT stereotaxy. ${ }^{13}$ In the current study, there was no significant difference in the success rate in treatment with or without USG. However, there could be selection bias as free-hand trocar insertion for ETV would be performed in less complicated cases without the use of USG.

The role of ETV for shunt infection was reported in 4 children; it allowed removal of all VP shunt implants. ${ }^{11}$ Subsequently, Shimizu et al. reported the use of ETV in 9 children with infected shunt as a bridging therapy and 7 of the 9 children required reinsertion of VP shunt after ETV. ${ }^{24}$ History of infection was shown to be an independent risk factor for ETV failure in cases of obstructive hydrocephalus. ${ }^{7}$ Our study, which included both communicating and obstructive hydrocephalus, has demonstrated that a history of infection is an independent risk factor for ETV failure in treating blocked shunts.

\section{Conclusions}

Emergency ETV had a comparable success rate to that of elective ETV. A history of CNS infection is an independent predictor of ETV failure in treating patients with blocked shunts.

\section{Acknowledgments}

We thank the neurosurgery residents at the University of Hong Kong, Queen Mary Hospital, for their contribution in the recruitment of patients and acquisition of data.

\section{References}

1. Bilginer B, Oguz KK, Akalan N: Endoscopic third ventriculostomy for malfunction in previously shunted infants. Childs Nerv Syst 25:683-688, 2009

2. Boschert J, Hellwig D, Krauss JK: Endoscopic third ventriculostomy for shunt dysfunction in occlusive hydrocephalus: long-term follow up and review. J Neurosurg 98:1032-1039, 2003

3. Chan DYC, Zhu CXL, Chan DTM, Poon WS: Extraventricular intracisternal obstructive hydrocephalus? A differential diagnosis of "communicating hydrocephalus" after a successful endoscopic third ventriculostomy. Surg Pract 22:145147, 2018

4. Chhun V, Sacko O, Boetto S, Roux FE: Third ventriculocisternostomy for shunt failure. World Neurosurg 83:970-975, 2015

5. Cinalli G, Salazar C, Mallucci C, Yada JZ, Zerah M, SainteRose C: The role of endoscopic third ventriculostomy in the management of shunt malfunction. Neurosurgery 43:13231329, 1998

6. Drake JM: Endoscopic third ventriculostomy in pediatric patients: the Canadian experience. Neurosurgery 60:881-886, 2007
7. Fukuhara T, Vorster SJ, Luciano MG: Risk factors for failure of endoscopic third ventriculostomy for obstructive hydrocephalus. Neurosurgery 46:1100-1111, 2000

8. Greitz D: Paradigm shift in hydrocephalus research in legacy of Dandy's pioneering work: rationale for third ventriculostomy in communicating hydrocephalus. Childs Nerv Syst 23:487-489, 2007

9. Hailong F, Guangfu H, Haibin T, Hong P, Yong C, Weidong L, et al: Endoscopic third ventriculostomy in the management of communicating hydrocephalus: a preliminary study. J Neurosurg 109:923-930, 2008

10. Hopf NJ, Grunert P, Fries G, Resch KDM, Perneczky A: Endoscopic third ventriculostomy: outcome analysis of 100 consecutive procedures. Neurosurgery 44:795-806, 1999

11. Jones RFC, Stening WA, Kwok BCT, Sands TM: Third ventriculostomy for shunt infections in children. Neurosurgery 32:855-860, 1993

12. Kehler U, Gliemroth J: Extraventricular intracisternal obstructive hydrocephalus - a hypothesis to explain successful 3 rd ventriculostomy in communicating hydrocephalus. Pediatr Neurosurg 38:98-101, 2003

13. Kelly PJ: Stereotactic third ventriculostomy in patients with nontumoral adolescent/adult onset aqueductal stenosis and symptomatic hydrocephalus. J Neurosurg 75:865-873, 1991

14. Kita D, Hayashi Y, Fukui I, Oishi M, Nakada M: Simultaneous ventriculoperitoneal shunt removal and endoscopic third ventriculostomy for three patients previously treated for intracranial germ cell tumors more than 20 years ago. Childs Nerv Syst 32:1543-1547, 2016

15. Kulkarni AV, Drake JM, Kestle JRW, Mallucci CL, Sgouros S, Constantini S: Predicting who will benefit from endoscopic third ventriculostomy compared with shunt insertion in childhood hydrocephalus using the ETV Success Score. J Neurosurg Pediatr 6:310-315, 2010

16. Kulkarni AV, Drake JM, Mallucci CL, Sgouros S, Roth J, Constantini S: Endoscopic third ventriculostomy in the treatment of childhood hydrocephalus. J Pediatr 155:254-259, 259.e1, 2009

17. Kulkarni AV, Riva-Cambrin J, Browd SR: Use of the ETV Success Score to explain the variation in reported endoscopic third ventriculostomy success rates among published case series of childhood hydrocephalus. J Neurosurg Pediatr 7:143-146, 2011

18. Marton E, Feletti A, Basaldella L, Longatti P: Endoscopic third ventriculostomy in previously shunted children: a retrospective study. Childs Nerv Syst 26:937-943, 2010

19. Naftel RP, Reed GT, Kulkarni AV, Wellons JC: Evaluating the Children's Hospital of Alabama endoscopic third ventriculostomy experience using the Endoscopic Third Ventriculostomy Success Score: an external validation study. J Neurosurg Pediatr 8:494-501, 2011

20. Neils DM, Wang H, Lin J: Endoscopic third ventriculostomy for shunt malfunction: what to do with the shunt? Surg Neurol Int 4:3, 2013

21. Nishiyama K, Mori H, Tanaka R: Changes in cerebrospinal fluid hydrodynamics following endoscopic third ventriculostomy for shunt-dependent noncommunicating hydrocephalus. J Neurosurg 98:1027-1031, 2003

22. O'Brien DF, Javadpour M, Collins DR, Spennato P, Mallucci CL: Endoscopic third ventriculostomy: an outcome analysis of primary cases and procedures performed after ventriculoperitoneal shunt malfunction. J Neurosurg 103 (5 Suppl):393-400, 2005

23. Rieger A, Rainov NG, Sanchin L, Schöpp G, Burkert W: Ultrasound-guided endoscopic fenestration of the third ventricle in obstructive hydrocephalus, in Hellwig D, Bauer BL (eds): Minimally Invasive Techniques for Neurosurgery. Berlin: Springer, 1998, pp 81-85

24. Shimizu T, Luciano MG, Fukuhara T: Role of endoscopic 
third ventriculostomy at infected cerebrospinal fluid shunt removal. J Neurosurg Pediatr 9:320-326, 2012

25. Spennato P, Ruggiero C, Aliberti F, Nastro A, Mirone G, Cinalli G: Third ventriculostomy in shunt malfunction.

World Neurosurg 79 (2 Suppl):22.e21-22.e26, 2013

\section{Disclosures}

The authors report no conflict of interest concerning the materials or methods used in this study or the findings specified in this paper.

\section{Author Contributions}

Conception and design: Ho, Chan, Cheng, Leung. Acquisition of data: Chan, ACO Tsang, Cheng, Li, FCP Tsang, Taw, Pu, Lui. Analysis and interpretation of data: Chan, ACO Tsang. Drafting the article: Chan. Critically revising the article: all authors.
Reviewed submitted version of manuscript: all authors. Approved the final version of the manuscript on behalf of all authors: Ho. Statistical analysis: Ho, Chan, ACO Tsang, Leung, Lui. Administrative/technical/material support: Ho, Cheng, Li, FCP Tsang, Taw, Pu, Leung, Lui. Study supervision: Ho, Leung, Lui.

\section{Supplemental Information}

\section{Previous Presentations}

Part of this paper was previously presented as an oral presentation at the Hong Kong Neurosurgical Society Monthly Academic Meeting on September 13, 2017, at Queen Elizabeth Hospital, Jordon, Hong Kong.

\section{Correspondence}

Wilson W. S. Ho: The University of Hong Kong, Queen Mary Hospital, Hong Kong. howsw@hku.hk. 